\title{
Analysis of the mitochondrial maxicircle of Trypanosoma lewisi, a neglected human pathogen
}

Ruo-Hong Lin ${ }^{1 \dagger}$, De-Hua Lai ${ }^{1{ }^{*}}$, Ling-Ling Zheng ${ }^{2}$, Jie Wu ${ }^{2}$, Julius Lukeš 3,4 , Geoff Hide ${ }^{5}$ and Zhao-Rong Lun ${ }^{1,2,5^{*}}$

\begin{abstract}
Background: The haemoflagellate Trypanosoma lewisi is a kinetoplastid parasite which, as it has been recently reported to cause human disease, deserves increased attention. Characteristic features of all kinetoplastid flagellates are a uniquely structured mitochondrial DNA or kinetoplast, comprised of a network of catenated DNA circles, and RNA editing of mitochondrial transcripts. The aim of this study was to describe the kinetoplast DNA of T. lewisi.

Methods/Results: In this study, purified kinetoplast DNA from T. lewisi was sequenced using high-throughput sequencing in combination with sequencing of PCR amplicons. This allowed the assembly of the T. lewisi kinetoplast maxicircle DNA, which is a homologue of the mitochondrial genome in other eukaryotes. The assembly of 23,745 bp comprises the non-coding and coding regions. Comparative analysis of the maxicircle sequence of $T$. lewisi with Trypanosoma cruzi, Trypanosoma rangeli, Trypanosoma brucei and Leishmania tarentolae revealed that it shares $78 \%, 77 \%, 74 \%$ and $66 \%$ sequence identity with these parasites, respectively. The high GC content in at least 9 maxicircle genes of T. lewisi (ATPase6; NADH dehydrogenase subunits ND3, ND7, ND8 and ND9; G-rich regions GR3 and GR4; cytochrome oxidase subunit COIII and ribosomal protein RPS12) implies that their products may be extensively edited. A detailed analysis of the non-coding region revealed that it contains numerous repeat motifs and palindromes.

Conclusions: We have sequenced and comprehensively annotated the kinetoplast maxicircle of T. lewisi. Our analysis reveals that $T$. lewisi is closely related to T. cruzi and T. brucei, and may share similar RNA editing patterns with them rather than with $L$. tarentolae. These findings provide novel insight into the biological features of this emerging human pathogen.
\end{abstract}

Keywords: Trypanosoma lewisi, Kinetoplast maxicircle, Mitochondrial DNA, RNA editing, Palindrome

\section{Background}

The genus Trypanosoma belongs to the Kinetoplastea, which lies within the eukaryotic supergroup Excavata and comprises an assembly of mostly parasitic flagellated protists [1]. The best known trypanosomes are the human pathogenic Trypanosoma brucei gambiense and $T$. $b$. rhodesiense causing sleeping sickness in Africa, and $T$. cruzi, the causative agents of Chagas disease in South

\footnotetext{
* Correspondence: laidehua@mail.sysu.edu.cn; Isslzr@mail.sysu.edu.cn

${ }^{\dagger}$ Equal contributors

${ }^{1}$ Center for Parasitic Organisms, State Key Laboratory of Biocontrol, School of Life Sciences and Key Laboratory of Tropical Diseases and Control of the Ministry of Education, Zhongshan School of Medicine, Sun Yat-Sen University, Guangzhou, The People's Republic of China

Full list of author information is available at the end of the article
}

America. Other members of this genus are economically important animal parasites. Trypanosoma lewisi has long been recognized as a globally distributed obligatory parasite of rodents of the genus Rattus, transmitted by rat fleas and non-pathogenic to its natural hosts and humans [2]. This view has changed recently when human infections were reported [3] which culminated with a case of a fatal infection in an infant with a T. lewisi-like flagellate [4]. More importantly, the resistance of this parasite to the lysis by normal human serum was recently demonstrated [5]. These reports substantially raise the importance of this flea-transmitted trypanosome, which can now be considered a neglected human parasite $[3,6-8]$. This is particularly important in developing countries where infants may have encountered direct 
contact with T. lewisi-infected rat fleas. More research on $T$. lewisi is warranted to establish a greater knowledge of the basic biology of this organism and its potential for contribution to human disease.

As a trypanosomatid flagellate, T. lewisi has an extensive mitochondrial DNA network composed of mutually interlocking DNA circles which are packed into a diskshaped structure termed the kinetoplast DNA (kDNA). The kDNA consists of dozens of catenated maxicircles with species-specific sizes, ranging from 20 to $40 \mathrm{~kb}$, and thousands of minicircles ranging from 0.5 to $10 \mathrm{~kb}$, again in a species-specific manner [9-11]. The kDNA maxicircle is composed of two regions: a coding region carrying homologs of mitochondrial genes typical of other eukaryotes $[12,13]$ and a variable non-coding region, also known as the divergent region (DR), which may play a role in maxicircle replication [14]. The coding region contains two ribosomal RNA genes, fourteen protein-coding genes (ND8, ND9, ND7, COIII, Cyb, ATPase6, MURF1 (now known to be ND2 [15]), ND1, COII, COI, ND4, ND3, RSP12, ND5), four genes (MURF2, MURF5, GR3 and GR4) of unknown function [16], and a few gRNAs (guide RNA) [16, 17]. The minicircles encode heterogenous guide RNA genes, which provide information for extensive RNA editing of maxicircle transcripts [18, 19]. This type of post-transcriptional modification entails the insertions, and less frequently deletions of uridines, using complex protein machinery and interactions [20-23].

Kinetoplast DNA has been established as a good taxonomic marker since it has a relatively fast rate of evolution [24]. Moreover, it is key to our understanding of RNA editing patterns and overall mitochondrial function. Hence, alterations in the kDNA may have a substantial impact on parasite development and the course of infection. It has been reported that $T$. cruzi isolates carrying a major deletion in the maxicircle-encoded ND7 gene seem to cause only asymptomatic Chagas disease [25]. Furthermore, depletions and losses of the kDNA have been shown to play an important role in the evolution of trypanosomes, leading to the emergence of new species that are able to occupy new niches [26-28]. Therefore, the kDNA has a significant impact on the transmission, pathogenicity, development and evolution of trypanosomes.

Despite its potential importance as a zoonotic pathogen, no information is available on the maxicircle kDNA of $T$. lewisi [5]. Here, we present a well-annotated maxicircle sequence of the Chinese strain of T. lewisi. Comparative analyses with the maxicircle sequences of $T$. brucei, $T$. cruzi, $T$. rangeli and Leishmania tarentolae revealed high overall conservation of gene content and synteny. Our work provides a framework for future studies of the kDNA biology, RNA editing and evolution of T. lewisi. In addition, genetic information on the T. lewisi maxicircle can be used to design diagnostic molecular markers needed for the detection of this parasite in natural hosts as well as in humans.

\section{Methods}

Parasites, ultrastructure, isolation of kDNA and restriction endonuclease digestion

Trypanosoma lewisi CPO02 strain, isolated from a rat (Rattus norvegicus) trapped in Guangzhou [7], was used in this study. Trypanosomes were grown in Sprague Dawley rats and were harvested from blood by differential centrifugation. Briefly, red blood cells were pelleted at 180 $\mathrm{x} g$ for $10 \mathrm{~min}$, flagellates were carefully transferred from the supernatant to a new tube and spun at $1500 \mathrm{x} g$ for $5 \mathrm{~min}$. For transmission electron microscopy, specimens were prepared following a protocol described elsewhere [29] and observed under a JEM-100CX-II microscope system. The kDNA networks were isolated by sucrose gradient ultracentrifugation using a previously described protocol [30]. The isolated $\mathrm{kDNA}$ were visualized on $1 \%$ agarose gel. Endonucleases MspI, MboI, BamHI, TaqI, HindIII, RsaI, and HaeIII (New England Biolabs, USA) were used for restriction enzyme analysis and were carried out according to the conditions recommended by the manufacturer. Computer-simulated restriction enzyme digestion map of T. lewisi maxicircle was performed using the Vector NTI software suite [31].

\section{Deep sequencing, assembly, PCR verification}

A kDNA library of $T$. lewisi was commercially prepared and $100 \mathrm{bp}$-long paired-end reads were obtained by Illumina Hiseq 2000 (Novogene, China) and assembled into contigs by Velvet version 1.2.10 software [32]. Maxicircle contigs were identified by alignment with published maxicircles of T. cruzi (GenBank: DQ343645), T. rangeli (GenBank: KJ803830.1), T. brucei (GenBank: M94286.1) and L. tarentolae (GenBank: M10126.1) using NCBI BLAST software. The assembly of contigs was confirmed and gaps between contigs (NODE_60 and NODE_165; NODE_165 and NODE_28) were filled-in by sequencing PCR products obtained with 11 pairs of primers, listed in Table 1. In order to obtain the sequence of the noncoding DR between the contigs NODE_28 and NODE_60, a forward primer adapted from the 3' end of NODE_28 and two reverse primers from the $5^{\prime}$ end of NODE_60 were used for PCR amplifications. A commercial kit (Prime STAR Max DNA Polymerase, TaKaRa, China) was utilized for all PCR amplifications.

\section{Data analysis}

Alignment and manual annotation of $T$. lewisi sequences were carried out by comparison with other available maxicircle sequences, including maxicircles of $T$. cruzi strain Esmeraldo (GenBank: DQ343646.1), T. cruzi strain 
Table 1 Primers for PCR amplification of the T. lewisi maxicircle

\begin{tabular}{|c|c|c|}
\hline Primer & Sequence $\left(5^{\prime} \rightarrow 3^{\prime}\right)$ & Fragment position $^{a}$ \\
\hline TI Frag1-F & GCTAATTGCACTAATCGAGGT & $(-552)-1446$ \\
\hline TI Frag1-R & GCTGGCATCCATTTCTGACT & \\
\hline TI Frag2-F & AAAGGTCCGAGCAGGTTA & $977-3001$ \\
\hline TI Frag2-R & CTITCTGTGCCACGATGT & \\
\hline TI Frag3-F & ATAAGAATAAGAGGGACAAACC & $2877-4831$ \\
\hline TI Frag3-R & CGCATCTGAACTCATAAAATAG & \\
\hline TI Frag4-F & AGGTITTGTAGTGCGTAGTGTAC & $4446-5267$ \\
\hline TI Frag4-R & ATTCCATTCATATTGGATAAGC & \\
\hline Tl Frag5-F & TTTATTGTGAACGGTTATGCT & $4851-8008$ \\
\hline TI Frag5-R & ACAACTTCGGATTGGACCT & \\
\hline Tl Frag6-F & ATGGCTGCGAGATAAACAA & $7697-9586$ \\
\hline Tl Frag6-R & GGCATTAAAACAAAACAACTT & \\
\hline TI Frag7-F & TATTTGGATCATACGCCTTA & 8980-11526 \\
\hline TI Frag7-R & GGAATGATAAAGCGGGAA & \\
\hline TI Frag8-F & AAAATCCGCTAACTAAACACC & $11104-12847$ \\
\hline TI Frag8-R & CCTAAGAAAAGGGAACTTCATAC & \\
\hline TI Frag9-F & TATTTCTAATGGGGCTTGTG & 11971-13782 \\
\hline TI Frag9-R & CACAGAAATCGTAATAGCAATAC & \\
\hline TI Frag10-F & GGAAGTITACTITAGGAAGGC & $13644-14639$ \\
\hline Tl Frag10-R & GTGGATTCATACACCCATGAC & \\
\hline TI Frag11-F & GGAAGGACCAATCCCAGTT & 14306-16095 \\
\hline TI Frag11-R & TGTACGTTACAATTCGGTGTTT & \\
\hline TI DR-1 F & CCATTAAAACCAAATTAGGTG & $16855-(-2912)$ \\
\hline TI DR-1R & GGAGAGAAGGGAAAATAAGG & \\
\hline TI DR-2 F & CCATTAAAACCAAATTAGGTG & $16855-(-3218)$ \\
\hline TI DR-2R & TCGTATAAAGCGATGTGAAAG & \\
\hline
\end{tabular}

apresents fragment positions are shown relative to the start of the $12 \mathrm{~S} r R N A$, and the positions located before $12 S$ rRNA gene are indicated with minus signs and enclosed in parentheses

b presents sequences of primers TIDR-1 F and TIDR-2 F are same

marinkellei (GenBank: KC427240.1), T. cruzi strain Silvio (GenBank:FJ203996.1), T. equiperdum (GenBank: EU185800.1), T. congolense (Tritrypdb: T.congo_bin 13880417 to 13889953), T. vivax (GenBank: KM386508.1). Phylogenetic tree was constructed based on Neighbor joining or Maximum likelihood methods with 1,000 bootstrap replicates using MEGA 4.0 [33]. Dot matrix graphs of the T. lewisi maxicircle sequence against itself and maxicircles from other trypanosomatids were generated using EMBOSS software suite [34]. Artemis software was used to generate GC percentage graphs of the maxicircle coding region [35] and identity indices among trypanosomatid protists were calculated with BioEdit software [36]. Motifs in the DR sequence were identified and presented in LOGO diagrams by MEME software [37].

\section{Ethical approval}

In this study, rats were treated in strict accordance to the guidelines for Medical Laboratory Animals (1998) from Ministry of Health, China, under the protocols approved by National Institute for Communicable Disease Control and Prevention and Laboratory Animal Use and Care Committee of Sun Yat-Sen University under the licenses of 2010 CB53000.

\section{Results \\ Morphology, ultrastructure, kDNA isolation and restriction enzyme digestion}

In vertebrate blood, the predominant morphological type is the slender trypomastigote form in which a prominent kinetoplast, about one tenth of the size of the nucleus, is located close to the posterior end, as judged by Diff Quick staining (Fig. 1a). Ultrastructural analysis of this stage revealed that the $\mathrm{kDNA}$ disk measures $588 \pm$ $92 \mathrm{~nm}$ in length and $138 \pm 18 \mathrm{~nm}$ in width $(n=65)$ (Fig. 1b), which is similar to other trypanosomes.

A total of $\sim 10^{9} \mathrm{~T}$. lewisi bloodstream trypomastigotes were harvested from infected rats. High quality kDNA was obtained as judged by the $260 / 280$ absorbance ratio of T. lewisi kDNA which was 1.86 . To verify its quality, an aliquot of the kDNA sample was run on an agarose gel. The kDNA was intact and free from contamination of the nuclear and host DNAs, since no corresponding bands of nuclear DNA was found (See Additional file 1: Figure S1).

The restriction enzyme digestion pattern of the $T$. lewisi maxicircle was obtained by incubation with the endonucleases MspI, MboI, BamHI, TaqI, HindIII, RsaI and HaeIII. Following digestion, linearized fragments released from the catenated kDNA were separated by agarose gel electrophoresis and recorded (Additional file 2: Figure S2A). A high frequency of cleavages of kDNA minicircles was achieved by incubation with MboI, TaqI and HaeIII, as indicated by the released kDNA fragments. Most likely, all bands with molecular sizes of over $4 \mathrm{~kb}$ are derived from the kDNA maxicircles. The presence of some high molecular weight bands with a size greater than $20 \mathrm{~kb}$, found in the samples which were digested by MspI, BamHI, HindIII and HaeIII, indicate that the full-size kDNA maxicircle is larger than $20 \mathrm{~kb}$. The abundant bands smaller than $2.0 \mathrm{~kb}$ imply the presence of a high number of likely heterogeneous minicircles in the kDNA of T. lewisi. This is a good correlation with the disk-like ultrastructure of its kDNA (Fig. 1), which is clearly composed of thousands of densely packed minicircles.

\section{Assembly and annotation of the kDNA maxicircle}

To obtain the full-size maxicircle sequence, the purified kDNA was deep sequenced on the Illumina Hiseq 2000 

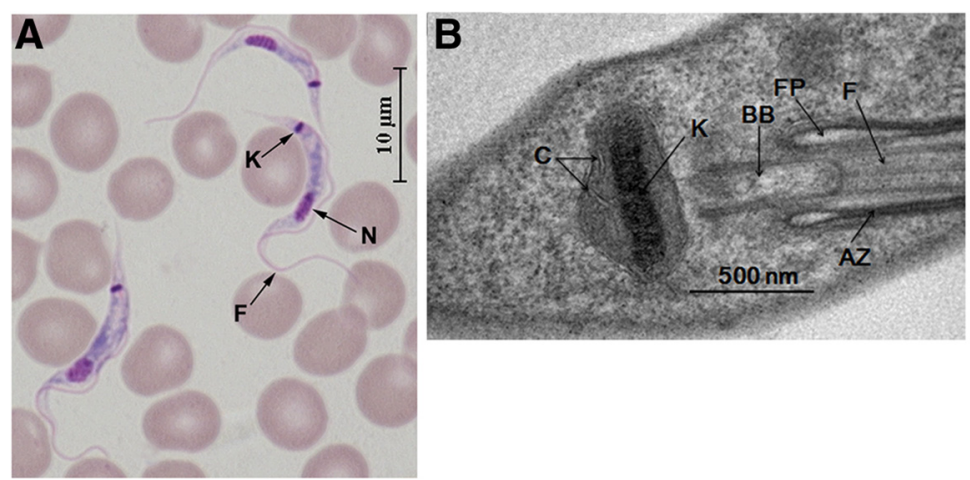

Fig. 1 Morphology and ultrastructure of T. lewisi. a Diff Quick staining of bloodstream forms of T. lewisi from rat. Flagellum (F), Nucleus (N) and Kinetoplast (K) are indicated. b Electron micrograph of T. lewisi trypomastigote form. Attachment Zone (AZ), Basic Body (BB), Mitochondrial Cristae $(\mathrm{MC})$, Flagellum (F), Flagellar Pocket (FP) and Kinetoplast (K) are indicated

platform. A total of 5,742,059 raw pair-end reads were generated. After removal of adapters, poly- $\mathrm{N}$ reads (reads containing $>10 \%$ poly-N) and low quality reads $(\mathrm{sQ}<=5)$ from raw data, we obtained 5,592,095 clean reads with Q20 (94.95\%), Q30 (87.64 \%) and GC content $(36.45 \%)$. These data were finally assembled into 528 contigs using the Velvet software. As expected, no host DNA sequences were found, confirming the high purity of the sample. Three T. lewisi maxicircle contigs (NODE_60, NODE_165, NODE_28) were identified by the BLAST search against the T. cruzi, T. rangeli, T. brucei and $L$. tarentolae maxicircle sequences. The rest of the contigs are likely to be the sequences from minicircles or divergent region of maxicircle. They have an average coverage of $35 \mathrm{x}$, which provides a high level of confidence for the assembly. These contigs were further verified by sequencing PCR amplicons obtained with 11 primer pairs (Additional file 3: Figure S3A), by which gaps within the coding region have been filled. A combination of three assembled contigs and 11 PCR amplicons thus produced a 20,618 bp-long sequence. The sequence covers the entire coding region $(15,369 \mathrm{bp})$ and part of the non-coding region $(5,249 \mathrm{bp})$ of the $T$. lewisi maxicircle, which is shown in Fig. 2.

To obtain the remaining non-coding DR sequence, two additional primer pairs (TIDR $1 \mathrm{~F} / 1 \mathrm{R}$ and TIDR $1 \mathrm{~F} / 2 \mathrm{R}$ ) from the contigs of NODE_28 and NODE_60 were designed and repeatedly used in PCR reactions (Additional file 3: Figure S3B). However, due to numerous repetitions in this region, we were unable to assemble the whole region. Accordingly, only 3,127-bp of new sequence derived from this region was obtained. Another BLAST search using this new sequence as a query identified an additional set of contigs (NODE_805, NODE_1689, NODE_1919, NODE_587, NODE_302). Altogether, a total of 23,745 bp of the T. lewisi maxicircle was obtained and deposited in the GenBank (accession no. KR072974).
According to previous reports [12, 38], with the exception of some pan-edited regions, the nucleotide sequences and gene order of the maxicircle coding region are highly conserved among trypanosomatid flagellates, allowing a straightforward annotation of the T. lewisi kDNA maxicircle. Our data indicate that its maxicircle encodes 20 tightly clustered genes (Table 2), with a gene order syntenic with that of T. cruzi, T. brucei and L. tarentolae. We have also launched a computer-simulated virtual restriction digestion and electrophoresis (VRDE) to evaluate the physical map of this assembled maxicircle (Additional file 2: Figure S2B). Since VRDE considers only complete cleavage by restriction enzymes, it predicted at least 42 restriction sites for RsaI and TaqI, butonly several target sites for HindIII, HaeIII and MspI, and just one for BamHI. All computer-simulated restriction fragments which were greater than $4.0 \mathrm{~kb}$ from the

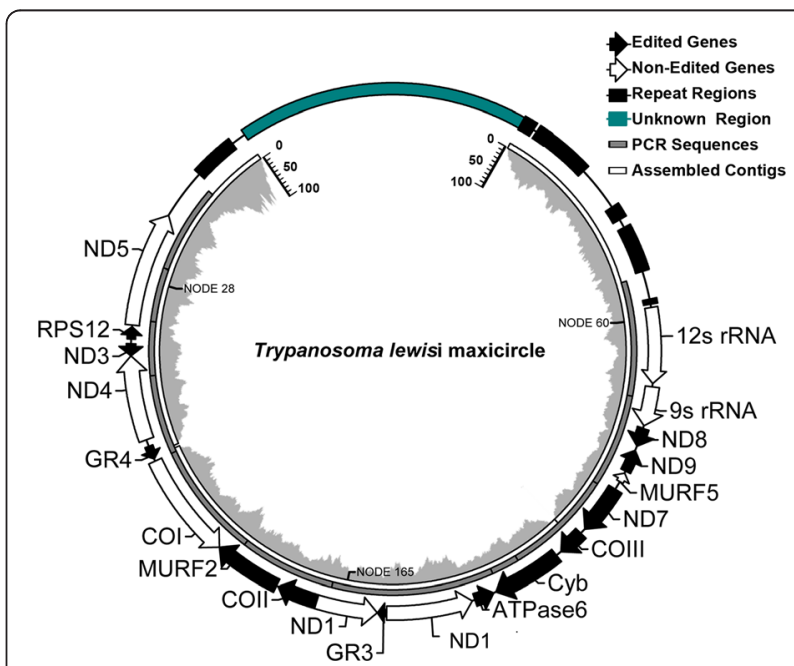

Fig. 2 Diagram of the T. lewisi maxicircle. The diagram is composed of fourloops, from inner to outer are assembly coverage, assembled contigs, PCR sequencing and geneorganization, respectively 
Table 2 Gene positions and lengths in the T. lewisi maxicircle

\begin{tabular}{|c|c|c|c|c|c|c|}
\hline Gene & RNA editing & T. lewisi position & T. lewisi length & T. cruzi length ${ }^{d}$ & T. brucei length ${ }^{e}$ & L. tarentolae lengthe \\
\hline $12 S$ rRNA & / & $1-1168$ & 1168 & 1161 & 1149 & 1173 \\
\hline $9 S$ rRNA & / & $1218-1825$ & 608 & 608 & 611 & 611 \\
\hline ND8 & Extensive & $1865-2149$ & 285 & 279 & 266 & 266 \\
\hline$D N 9^{a}$ & Extensive & $2218-2567$ & 350 & 338 & 321 & 291 \\
\hline$M U R F 5^{\mathrm{a}}$ & None & $2581-2821$ & 241 & 264 & 234 & 303 \\
\hline$N D 7^{\mathrm{b}}$ & Extensive & $2887-3657$ & 771 & 755 & 702 & 1144 \\
\hline COIII ${ }^{b}$ & Extensive & $3741-4154$ & 414 & 424 & 439 & 852 \\
\hline Cyb & Minor & $4242-5321$ & 1080 & 1080 & 1080 & 1079 \\
\hline ATPase $^{\mathrm{b}}$ & Extensive & 5359-5662 & 304 & 336 & 369 & 603 \\
\hline MURF1/(ND2) $)^{a}$ & None & $5704-7044$ & 1341 & 1341 & 1237 & 1332 \\
\hline$G R 3^{c}$ & Extensive & 7033-7155 & $\sim 123$ & $\sim 119$ & $\sim 164$ & 167 \\
\hline$N D 1^{\mathrm{a}}$ & None & $7172-8113$ & 942 & 942 & 957 & 942 \\
\hline COII & Minor & $8115-8743$ & 629 & 629 & 626 & 629 \\
\hline MURF2 & Minor & 8770-9822 & 1053 & 1056 & 1041 & 1045 \\
\hline $\mathrm{CO}^{\mathrm{A}}$ & None & $9813-11462$ & 1650 & 1650 & 1734 & 1650 \\
\hline$G R 4^{a}$ & Extensive & 11508-11719 & 212 & 207 & 185 & 189 \\
\hline ND4 & None & 11824-13137 & 1314 & 1314 & 1311 & 1314 \\
\hline$N D 3^{\mathrm{a}}$ & Extensive & 13126-13312 & 187 & 193 & 256 & 187 \\
\hline RPS12 & Extensive & 13383-13572 & 190 & 191 & 172 & 182 \\
\hline ND5 & None & 13597-15369 & 1773 & 1770 & 1770 & 1773 \\
\hline
\end{tabular}

Gene positions are shown relative to the start of the gene $12 \mathrm{~S}$ rRNA

apresents those genes that are encoded by the reverse strand

${ }^{b}$ presents those genes that are $5^{\prime}$-edited in $L$. tarentolae

cpresents the two end positions of GR3 from T. lewisi, T. cruzi and T. brucei are uncertain

${ }^{d}$ presents the maxicircle gene lengths of $T$. cruzi that are cited from the paper of Ruvalcaba-Trejo [16]

epresents calculations of the maxicircle gene lengths of T. brucei and L. tarentolae that are based on the data from GenBank (M94286.1 and

M10126.1, respectively)

23,745 bp-long sequence were experimentally verified by restriction digests (asterisks in Additional file 2: Figure S2B), confirming a high correlation between both approaches. However, a handful of weak bands appeared in agarose gels that were not predicted by the software (question marks in Additional file 2: Figure S2B) implying either that they originate from an unknown region or from an incomplete digestion.

\section{Comparative analysis of trypanosomatid maxicircles}

A dot matrix analysis was employed to compare the $T$. lewisi maxicircle with those of $T$. cruzi, $T$. brucei, T. rangeli and L. tarentolae (Additional file 4: Figure S4). Similar sequences between two species are indicated by diagonal lines, each dot represents a full identity over a $10 \mathrm{bp}$-long window. Based on the graphs, we can clearly see that the T. lewisi maxicircle displays higher overall sequence identity to $T$. cruzi, $T$. rangeli and $T$. brucei than to $L$. tarentolae. The sequences of coding regions and especially the region corresponding to the genes the transcripts of which do not undergo RNA editing (12S
rRNA, 9S rRNA, ND2, ND1, COI, ND4 and ND5) have a high degree of identity among all compared species, while much bigger differences were found in their Divergent Region (DR) sequences. Variations in the coding regions which caused shifts and breaks on the diagonal line occurred mainly in genes with transcripts subject to (extensive) editing, namely $N D 8, N D 9, N D 7$, COIII, ATPase6 and GR4. An array of substantial breaks appeared in the comparison with $L$. tarentolae (Additional file 4: Figure S4D, red box). RNA editing of ND7, COIII and ATPase6 in L. tarentolae were shown to be limited to the $5^{\prime}$ region but are extensive in the same mRNAs in T. brucei $[39,40]$. These breaks suggest that the editing patterns in $T$. lewisi may be more similar to other trypanosomes than to L. tarentolae. Moreover, the analysis of the maxicircle shows that it also carries guide RNA genes. This is judged by a guide RNA bearing information compatible with the editing of the COII mRNA. However, without validation at the RNA level, these guide RNAs have not yet been included in this annotation. 
GC plot and comparative analysis of maxicircle coding regions and mature mRNA predictions

The dot matrix comparison analysis mentioned above indicated that editing pattern in the T. lewisi maxicircle may be similar to those in $T$. cruzi and T. brucei. In order to further predict editing sites, we analyzed the GC content of the T. brucei maxicircle (Additional file 5: Figure S5A), as a reference whose RNA editing pattern is well documented, and the GC content of the T. lewisi maxicircle coding regions. Indeed, the corresponding regions of extensively edited T. brucei genes (ND8, ND9, ND7, COIII, ATPase6, GR3, GR4, ND3 and RPS12) have higher GC contents than the non-edited genes. Since the GC scatter plot of T. lewisi is similar to that of T. brucei (Additional file 5: Figure S5A and S5B), high GC content genes may produce pan-edited transcripts, while those of COII, Cyb and MURF2 seem to undergo only limited editing. The continuous open reading frames of the remaining 8 genes testify to the lack of editing.

Pairwise alignment was done to evaluate the level of identity in the entire coding sequences and the edited and non-edited genes (both nucleotide and amino acid sequences) between $T$. lewisi and four trypanosomatids (Table 3). The entire coding sequence of T. lewisi shows highest and lowest levels of identity to $T$. cruzi and $L$. tarentolae, respectively. Sequences not subject to editing exhibit a higher level of identity among species than the extensively edited ones. We would like to point out that high identity among species was not confined to the non-edited genes, but was also observed in the 5' edited genes, indicating the presence of conserved editing patterns in these genes.

\section{Sequence analysis of maxicircle divergent region}

A common theme of the maxicircle DRs is the presence of various repeat arrays, which is also the case for $T$. lewisi. Mutual maxicircle comparison of data from the dot matrix identified two distinct sections (I, II) in the DR, flanking either $12 S$ rRNA or ND5 (Fig. 3a). Section I is composed of short and highly repetitive units of about $100 \mathrm{bp}$ (Fig. 3b), while section II consists of several large duplications (Fig. 3d). Three motifs (motif 1a, motif $1 \mathrm{~b}$ and motif 2) were found in section I by MEME analysis (Fig. 3c). Although they showed no similarity with other trypanosomatid species, the high confidence E-value (all < 1.0e-016) of these motifs indicate their functionality, particularly for $\mathrm{A}_{5} \mathrm{C}$-element-containing motif $1 \mathrm{a}$. Detailed analysis recognized that section II has a series of tandem elements, namely $\alpha, \beta$, and their shorter version $\alpha$, and $\beta$, and non-repeated element $\gamma$ (Fig. 3d). Three AT-rich palindromes are present in the element $\alpha / \alpha$. In the first element $\alpha$, palindrome 1 is 34 bp long (AGGTTTTTAAAAATA TAAATATTTTTAAAAACCT), located 4,666 nt upstream of $12 \operatorname{SrRNA}$. In the second element $\alpha$, palindrome 2 is 28 bp long (TTTTTAAAAATATAAATATTTTTAAAAA) and 2,652 nt upstream of $12 \operatorname{SrR} N A$. Although the third element $\alpha$ was labeled as $\alpha$ ' due to limited deletions, it still contains a $34 \mathrm{bp}$ long palindrome (ATGTTTTTAAAAA TATA-TATATTTTTAAAAACCT), $879 \mathrm{nt}$ upstream of the same gene. Comparison of these three palindromes with the maxicircles of T. cruzi and T. rangeli enabled the identification of similar palindromes in these species (Fig. 3e). Conservation of these AT-rich palindromes testifies to their functionality.

\section{Maxicircle-base phylogenetic inference}

Mitochondrial DNA sequences are considered valuable markers for the inference of phylogenetic relationships [24]. Use of the entire maxicircle coding sequence would be superior to single gene-based phylogenies. To further investigate the genetic relationships among five trypanosomatid species, their maxicircle coding regions were aligned and used to build a neighbor-joining tree, which revealed that $T$. lewisi clusters with $T$. cruzi and $T$. rangeli with $100 \%$ confidence (Fig. 4). Although both $T$. rangeli and $T$. lewisi belong to the same subgenus Herpetosoma, $T$. rangeli is more closely related, with over $95 \%$ confidence, to T. cruzi than to T. lewisi. African trypanosomes $T$. brucei, $T$. congolense, T.vivax and $T$. equiperdum were present in the second clade and all belong to the salivarian subgroup. Not surprisingly, $L$.

Table 3 Average percentage identity among the maxicircle DNA from five trypanosomatid species

\begin{tabular}{lllllll}
\hline Comparison of T. lewisi & Entire coding region & $5^{\prime}$-editedgenes & Extensively-edited genes & rRNAs & Non-edited genes & AAs of Non-edited genes \\
\hline vs. T. cruzi & $78.0 \%$ & $82.0 \%$ & $74.2 \%$ & $83.5 \%$ & $79.8 \%$ & $82.7 \%$ \\
vs. T. rangeli & $77.2 \%$ & $82.8 \%$ & $73.8 \%$ & $84.5 \%$ & $79.3 \%^{\mathrm{a}}$ & $82.5 \%^{\mathrm{a}}$ \\
vs. T. brucei & $74.1 \%$ & $81.8 \%$ & $61.7 \%$ & $79.4 \%$ & $77.2 \%$ & $78.4 \%$ \\
vs. L. tarentolae & $66.4 \%$ & $77.6 \%$ & $50.8 \%{ }^{\mathrm{b}} \%$ & $78.4 \%$ & $75.5 \%$ & $74.4 \%$ \\
\hline
\end{tabular}

Entire coding region: starting from the $5^{\prime}$ end of $12 S$ rRNA to the $3^{\prime}$ end of ND5

5'-edited genes: Cyb, COII, MURF2

Extensively-edited genes: ND9, ND8, ND7, COIII, ATPase6, GR3, GR4, RPS12

Non-edited genes: MURF2/(ND2), ND1, COI, ND4, ND5

Proteins: MURF (ND2), ND1, ND4, ND5, COI

apresents gene ND1 was not included in the analysis of non-edited genes and proteins of $T$. rangeli due to the lack of complete sequence of this species

bresents gene COIII, ND7 and ATPase6 were not included in the analysis in L. tarentolae due to different RNA editing patterns 


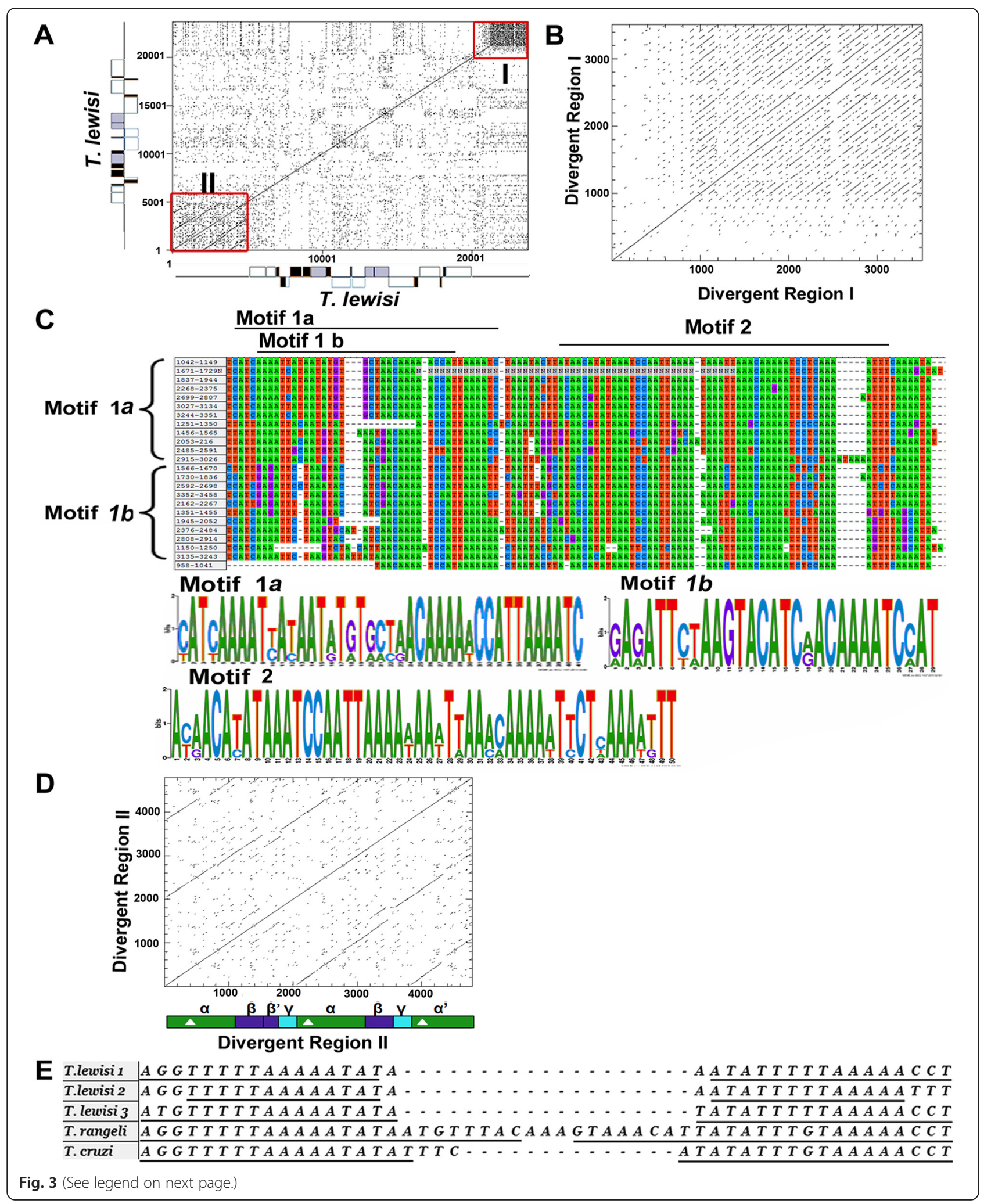


(See figure on previous page.)

Fig. 3 Sequence analyses of the T. lewisi maxicircle divergent region. Self Dottup graph of the T. lewisi entire maxicircle (a), DR section I (b), DR section II (d). Each dot represents an exact match over of $10 \mathrm{nt}$. Two distinct sections of DR region (I and II) are indicated in (a) with red boxes. The repeated elements $\left(a, \beta, \gamma, a^{\prime}\right.$ and $\left.\beta^{\prime}\right)$ identified from Dottup graph in (d) are illustrated, three palindromes within elements are indicated with white triangles. $\mathbf{c}$ The repetitive sequences from the section I were aligned and the position of three motifs is indicated with black line. LOGO diagrams show nucleotides at a given position of each motif and their relative frequency indicated by height. e The palindrome sequences from the DR of T. lewisi, T. rangeli and T. cruzi are shown with the inverted repeats underlined

tarentolae is separated from T. lewisi by a large genetic distance.

\section{Discussion}

Trypanosomatid protists including the pathogens causing leishmaniasis and trypanosomiasis in animals and humans invariably possess the uniquely structured kDNA [10, 41]. Since T. lewisi was recently defined as an opportunistic zoonotic and human blood parasite [5], it became particularly relevant to investigate its molecular and cellular features. Here we undertook an in-depth analysis of its maxicircle kDNA and compared it with its homologues in other trypanosomatids. We have combined deep sequencing and PCR in order to assemble, with sufficient sequence coverage, a $23.7 \mathrm{~kb}$-long region of the $T$. lewisi maxicircle. The estimated size of the complete maxicircle is approximately $24 \mathrm{~kb}$, which is within the size range of 20 to $40 \mathrm{~kb}$, estimated for other trypanosomatids studies thus far $[10,11]$.

The uridine insertion and/or deletion type of RNA editing is the most characteristic feature of the kinetoplastid mitochondrion. It is required to remove multiple frame shifts from about a dozen of transcripts, which encode subunits of respiratory complexes, rendering this post-transcriptional machinery essential $[20,22,42,43]$. Interestingly, both the set of transcripts and the extent of their editing are species-specific. For example, substantial differences in the editing patterns of ND7, COIII and ATPase6 have been documented; they are extensively edited in T. brucei, while only limited editing of the same transcripts occurs in L. tarentolae. Consequently, these so-called cryptogenes are much shorter in T. brucei [39]. Based both on the similarity of pre-edited gene sequences and the GC content plot of coding region, T. lewisi seems to have the $T$. cruzi-like editing pattern. However, this preliminary conclusion will have to be tested at the RNA level.

Even bigger inter-specific and inter-strain differences have been described for the maxicircle non-coding DR $[38,44,45]$. Its common feature is the presence of various repetitive sequences, which are also present in the DR of T. lewisi. Due to the absence of larger ORFs, it was proposed that this region may play a structural role and is also required for replication. This notion is based on the presence of a $12 \mathrm{bp}$-long conserved sequence block (CSB) in the DRs of T. brucei [46], Crithidia oncopelti [47] Leptomonas collosoma and Leptomonas seymouri [48]. CSB is a highly conserved replication origin of the kDNA minicircles, and it may fulfill the same role also in the maxicircles $[14,46]$. We attribute our failure to find CSB to its presence in the missing piece of the maxicircle DR of T. lewisi.

Furthermore, two palindromic elements were reported from the DRs, namely $39 \mathrm{bp}$ and about 40 bp-long palindromes in T. cruzi and Leishmania spp., respectively $[38,48]$. Although, these two palindromes were divergent in sequence, they both contain an $\mathrm{A}_{5} \mathrm{C}$-element, which reflects their putative association with transcription

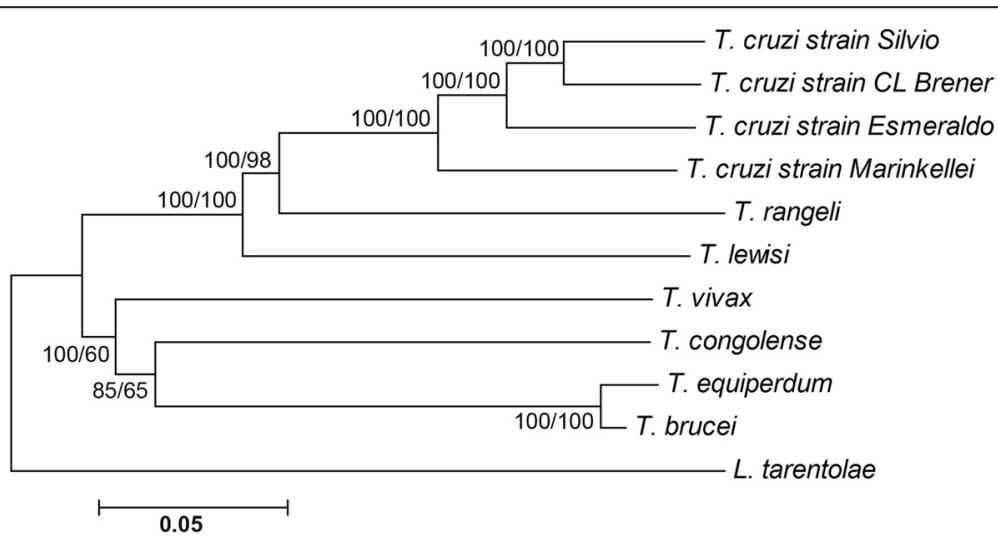

Fig. 4 Phylogenetic tree of maxicircle coding sequences from trypanosomatid species. Phylogenetic tree is performed based on Neighbor joining or Maximum likelihood methods with 1,000 bootstrap replicates. The scale bar represents the number of nucleotide substitutions per site. Partial T. congolense $(9 \mathrm{~kb}$ ) was retrieved from Tritrypdb by BLAST using other trypanosome maxicircle sequences 
initiation [49] or transcription factor binding sites [48]. In addition, palindromes may serve in a range of molecular capacities, such as the recognition sequences for restriction enzymes [50], binding sites for DNA-binding proteins [51] or may participate in control of gene expression [52]. As expected, we have identified three palindromes in the DR of $T$. lewisi, which are all homologous to the palindrome of T. cruzi. It is worth noting that we have also found a similar $53 \mathrm{bp}$-long palindrome sequence in the maxicircle DR of $T$. rangeli. All of these palindromes consist of the $\mathrm{A}_{5} \mathrm{C}$ element and two $\mathrm{T}_{5} \mathrm{~A}_{5}$ elements, implying these palindromes may play a significant role, yet their function remains unknown. Further functional research in $T$. lewisi and other kinetoplastids will be required to establish the role of these detailed sequence elements in the function and evolution of kinetoplasts and their DNA.

Mutual comparison with four members of the genus Trypanosoma and L. tarentolae using dot matrix comparison and average percentage identities revealed a high level of sequence similarity, with $T$. cruzi being more closely related. Our phylogenetic analysis also confirmed the well-established close relatedness of $T$. cruzi and $T$. lewisi. It is interesting to note that $T$. rangeli and $T$. lewisi are morphologically similar and both have been classified into the subgenus of Herpetosoma, while $T$. cruzi is affiliated with the subgenus Schizotrypanum [2]. However, T. rangeli is known to be quite different from others members of the genus Herpetosoma, both in terms of pathogenicity and transmission pathways, as well as based on the internal transcribed spacer and SSU rRNA sequences [7, 53, 54]. Hence, its reclassification into another subgenus has been suggested [53, 54], and our data from the mitochondrial genome lend further support for such a move.

The kinetoplast plays an important role in the life cycle of trypanosomes and, in particular, with respect to maintaining vector borne transmission cycles. A wide range of trypanosome species are found in an equally wide range of wild animal hosts globally. Typically, species of trypanosomes tend to have specific host/vector systems [2], such as the rat/rat flea with T. lewisi, discussed here, or other examples like the badger/badger flea in the case of $T$. pestanai $[55,56]$. Loss of parts of the kDNA have been demonstrated in trypanosome species, such as T. evansi and T. equiperdum, that may have escaped their traditional host vector systems [27]. These species are suggested to be only a few genetic steps away from the important human pathogen, T. brucei [28]. The discovery of human infections caused by atypical trypanosomes, such as T. evansi, T. congolense and T. lewisi $[3,8]$, raises concerns about the potential for human infectivity of many other trypanosomes with sylvatic cycles. Some trypanosome species, such as T. evansi and
T. equiperdum, have undergone an evolutionary adaptation to mechanical transmission between their specific hosts (e.g. camels and horses, respectively) without the need for developmental stages in a vector. The lack of kDNA maxicircles, found in T. evansi strains examined, is linked to a failure of key differentiation processes in $T$. brucei, which have been considered as an example of a cancer of parasitic protozoa [57]. More research is required to understand the role of $\mathrm{kDNA}$ in the evolution of diversity, transmission and pathogenicity within the kinetoplastids.

\section{Conclusions}

This is the first detailed analysis of the T. lewisi maxicircle. We show that it has a high level of similarity and the same gene order as other trypanosomatids. The most related species is, somewhat unexpectedly, T. cruzi. The predicted pattern of RNA editing is also quite similar to T. cruzi. A duplicated $\mathrm{A}_{5} \mathrm{C}$ element-containing palindrome was found in the DR of T. lewisi, suggesting its functional conservation. The sequence obtained from this neglected human pathogen provides information suitable for generation of a diagnostic assay.

\section{Additional files}

Additional file 1: Figure S1. Genome DNA and kinetoplast DNA preparations from T. lewisi were resolved on a 1\% agarose gel. Genome DNA and kinetoplast DNA preparations from T. lewisi were resolved on a 1\% agarose gel. Lanes: M, Molecular marker DL10000 (TaKaRa, Dalian, China); gDNA, genome DNA; kDNA, kinetoplast DNA. (PDF 88 kb)

Additional file 2: Figure S2. Restriction endonuclease analysis of $T$. lewisi kDNA. A) Ethidium Bromide-stained agarose gel showing restriction endonuclease digestion of $T$. lewisi kDNA with seven restriction enzymes. Marker, DL10000 (TaKaRa, Dalian, China). Note the presence of the kDNA network in some slots, indicating integrity of its network. B) Computersimulated virtual restriction patterns derived from the $23745 \mathrm{bp}$ T. lewisi maxicircle with the same set of restriction sites from (A). The larger, $>4.0$ kb-long fragments which were identified in (A), were marked with asterisks. A few weak bands over $4.0 \mathrm{~kb}$ in length in (A), which were not predicted by the software, were labeled with question marks. (PDF 144 kb)

Additional file 3: Figure S3. PCR amplification of $T$. lewisi maxicircles. A) Verification of the maxicircle coding region by PCR amplification using 11 pairs of primers, as visualized on a $1.0 \%$ agarose gel. Marker, DL10000 (TaKaRa, Dalian, China). B) A $1.0 \%$ agarose gel displaying PCR amplicons from the maxicircle divergent region obtained with two primer pairs. The 1F/1R, amplicon with TIDR 1F/1R primers, and the 1F/2R, amplicon with TIDR 1F/2R primers. Marker, 1 kb DNA Ladder (TIANGEN, China) and DL10000 (TaKaRa, Dalian, China). (PDF 142 kb)

Additional file 4: Figure S4. Dottup plot comparative analysis of maxicircle sequence of $T$. lewisi against maxicircle sequences of $T$. cruzi (A), T. rangeli (B), T. brucei (C) and L. tarentolae (D), respectively. Diagonal lines indicate that the DNA sequences of two compared species are identical in the corresponding regions. Each dot represents an exact match over of $10 \mathrm{nt}$. A remarkable break region in T. lewisi vs. L. tarentolae is indicated by a red box. (PDF $339 \mathrm{~kb}$ )

Additional file 5: Figure S5. Graphs show the GC percentage of $T$. brucei $(A)$ and $T$. lewisi (B) maxicircle coding regions. The regions where the percentage GC content value lies above the dashed lines are likely to undergo RNA editing. The window size used for this analysis was100 nt. (PDF 193 kb) 


\section{Abbreviations}

CO: Cytochrome oxidase; CSB: Conserved sequence block; GR: G-rich region; Cyb: Apocytochrome b; DR: Divergent region; gRNA: Guide RNA; ITS: Internal transcribed spacer; kDNA: Kinetoplast DNA; MURF: Mitochondrial unidentified reading frame; ND: NADH dehydrogenase; ORF: Open reading frame; RPS12: Ribosomal protein S12.

\section{Competing interests}

No competing interests are relevant to this article.

\section{Authors' contributions}

ZRL, DHL and RHL designed the study. RHL and DHL performed the experimental work. LLZ and JW carried out sequence assembly. RHL, DHL, $L L Z, J L, G H$ and ZRL analyzed the data. RHL, DHL, GH, and ZRL wrote the manuscript. All coauthors contributed to the editing of the text and approved the final manuscript.

\section{Acknowledgements}

We appreciate the kind assistance of members of the Center for Parasitic Organisms and Monica Namayanja. We acknowledge kind help of the Guangdong Province Key Laboratory of Computational Science and the Guangdong Province Computational Science Innovative Research Team. This work was supported by grants from the National Natural Science Foundation of China Grants (31472058, 31402029 and 31401975), Guangzhou Science Technology and Innovation Commission (201506010011) and the Natural Science Foundation of Guangdong Province (2014A030313164 and 2015A030311042)

\section{Author details}

'Center for Parasitic Organisms, State Key Laboratory of Biocontrol, School of Life Sciences and Key Laboratory of Tropical Diseases and Control of the Ministry of Education, Zhongshan School of Medicine, Sun Yat-Sen University, Guangzhou, The People's Republic of China. ${ }^{2}$ Key Laboratory of Gene Engineering of the Ministry of Education, State Key Laboratory of Biocontrol, School of Life Sciences, Sun Yat-Sen University, Guangzhou 510275, The People's Republic of China. Institute of Parasitology, Biology Centre, Czech Academy of Sciences and Faculty of Science, University of South Bohemia, České Budějovice (Budweis), Czech Republic. ${ }^{4}$ Canadian Institute for Advanced Research, Toronto, Canada. ${ }^{5}$ Ecosystems and Environment Research Centre and Biomedical Research Centre, School of Environment and Life Sciences, University of Salford, Salford, UK.

\section{Received: 23 November 2015 Accepted: 21 December 2015} Published online: 30 December 2015

\section{References}

1. Adl SM, Simpson AG, Lane CE, Lukeš J, Bass D, Bowser SS, et al. The revised classification of eukaryotes. J Eukaryot Microbiol. 2012;59:429-93.

2. Hoare CA. The trypanosomes of mammals: A zoological monograph. Oxford: Blackwell Scientific Publications; 1972.

3. Lun ZR, Reid SA, Lai DH, Li FJ. Atypical human trypanosomiasis: a neglected disease or just an unlucky accident? Trends Parasitol. 2009;25:107-8.

4. Verma A, Manchanda S, Kumar N, Sharma A, Goel M, Banerjee PS, et al. Trypanosoma lewisi or T. lewisi-like infection in a 37-day-old Indian infant. Am J Trop Med Hyg. 2011;85:221-4.

5. Lun ZR, Wen YZ, Uzureau P, Lecordier L, Lai DH, Lan YG, et al. Resistance to normal human serum reveals Trypanosoma lewisi as an underestimated human pathogen. Mol Biochem Parasitol. 2015;199:58-61.

6. de Sousa MA. On opportunist infections by Trypanosoma lewisi in humans and its differential diagnosis from T. cruzi and T. rangeli. Parasitol Res. 2014;113:4471-5.

7. Tang HJ, Lan YG, Wen YZ, Zhang XC, Desquesnes M, Yang TB, et al. Detection of Trypanosoma lewisi from wild rats in Southern China and its genetic diversity based on the ITS1 and ITS2 sequences. Infect Genet Evol. 2012;12:1046-51

8. Truc P, Buscher P, Cuny G, Gonzatti Ml, Jannin J, Joshi P, et al. Atypical human infections by animal trypanosomes. PLoS Negl Trop Dis. 2013;7, e2256.

9. Benne R. Mitochondrial genes in trypanosomes. Trends Genet. 1985;1:117-21.

10. Jensen RE, Englund PT. Network news: the replication of kinetoplast DNA. Ann Rev Microbiol. 2012;66:473-91.

11. Lukeš J, Guilbride DL, Votýpka J, Zíková A, Benne R, Englund PT. Kinetoplast DNA network: evolution of an improbable structure. Eukaryot Cell. 2002;1:495-502.
12. Simpson L, Neckelmann N, de la Cruz VF, Simpson AM, Feagin JE, Jasmer $\mathrm{DP}$, et al. Comparison of the maxicircle (mitochondrial) genomes of Leishmania tarentolae and Trypanosoma brucei at the level of nucleotide sequence. J Biol Chem. 1987;262:6182-96.

13. Stuart K. Kinetoplast DNA, mitochondria DNA with a difference. Mol Biochem Parasitol. 1983:9:93-104

14. Myler PJ, Glick D, Feagin JE, Morales TH, Stuart KD. Structural organization of the maxicircle variable region of Trypanosoma brucei: identification of potential replication origins and topoisomerase II binding sites. Nucl Acids Res. 1993;21:687-94.

15. Kannan S, Burger G. Unassigned MURF1 of kinetoplastids codes for NADH dehydrogenase subunit 2. BMC Genomics. 2008;9:455.

16. Ruvalcaba-Trejo LI, Sturm NR. The Trypanosoma cruzi Sylvio X10 strain maxicircle sequence: the third musketeer. BMC Genomics. 2011;12:58.

17. Blum B, Bakalara N, Simpson L. A model for RNA editing in kinetoplastid mitochondria: RNA molecules transcribed from maxicircle DNA provide the edited information. Cell. 1990:60:189-98.

18. Pollard WW, Rohrer SP, Michelotti EF, Hancock K, Hajduk SL. Organization of minicircle genes for guide RNAs in Trypanosoma brucei. Cell. 1990;63:783-90.

19. Sturm NR, Simpson L. Kinetoplast DNA minicircles encode guide RNAs for editing of cytochrome oxidase subunit III mRNA. Cell. 1990;61:879-84.

20. Lukeš J, Hashimi H, Zíková A. Unexplained complexity of the mitochondrial genome and transcriptome in kinetoplastid flagellates. Curr Genet. 2005:48:277-99

21. Simpson L, Sbicego S, Aphasizhev R. Uridine insertion/deletion RNA editing in trypanosome mitochondria: A complex business. RNA. 2003;9:265-76.

22. Verner Z, Basu S, Benz C, Dixit S, Dobáková EFD, Hashimi H, et al. Malleable mitochondrion of Trypanosoma brucei. Int Rev Cell Mol Biol. 2015;315:73-151.

23. Alfonzo JD, Thiemann O, Simpson L. The mechanism of $U$ insertion/deletion RNA editing in kinetoplastid mitochondria. Nucl Acids Res. 1997;25:3571-759.

24. Messenger LA, Llewellyn MS, Bhattacharyya T, Franzen O, Lewis MD, Ramirez $J D$, et al. Multiple mitochondrial introgression events and heteroplasmy in Trypanosoma cruzi revealed by maxicircle MLST and next generation sequencing. PLoS Negl Trop Dis. 2012;6, e1584.

25. Baptista CS, Vencio RZ, Abdala S, Carranza JC, Westenberger SJ, Silva MN, et al. Differential transcription profiles in Trypanosoma cruzi associated with clinical forms of Chagas disease: Maxicircle NADH dehydrogenase subunit 7 gene truncation in asymptomatic patient isolates. Mol Biochem Parasitol. 2006;150:236-48.

26. Carnes J, Anupama A, Balmer O, Jackson A, Lewis M, Brown R, et al. Genome and phylogenetic analyses of trypanosoma evansi reveal extensive similarity to T. brucei and multiple independent origins for dyskinetoplasty. PLoS Negl Trop Dis. 2015;9:e3404

27. Lai DH, Hashimi H, Lun ZR, Ayala FJ, Lukeš J. Adaptations of Typanosoma brucei to gradual loss of kinetoplast DNA: Trypanosoma equiperdum and Trypanosoma evansi are petite mutants of T. brucei. Proc Natl Acad Sci U S A. 2008;105:1999-2004

28. Lun ZR, Lai DH, Li FJ, Lukeš J, Ayala FJ. Trypanosoma brucei: two steps to spread out from Africa. Trends Parasitol. 2010;26:424-7.

29. Bozzola JJ. Conventional specimen preparation techniques for transmission electron microscopy of cultured cells. Meth Mol Biol. 2007;369:1-18.

30. Perez-Morga D, Englund PT. The structure of replicating kinetoplast DNA networks. J Cell Biol. 1993:123:1069-79.

31. Lu G, Moriyama EN. Vector NTI, a balanced all-in-one sequence analysis suite. Brief Bioinform. 2004;5:378-88.

32. Zerbino DR, Birney E. Velvet: algorithms for de novo short read assembly using de Bruijn graphs. Genome Res. 2008;18:821-9.

33. Kumar S, Nei M, Dudley J, Tamura K. MEGA: a biologist-centric software for evolutionary analysis of DNA and protein sequences. Brief Bioinform. 2008;9:299-306.

34. Rice P, Longden I, Bleasby A. EMBOSS: the European molecular biology open software suite. Trends Genet. 2000;16:276-7.

35. Carver T, Berriman M, Tivey A, Patel C, Bohme U, Barrell BG, et al. Artemis and $A C T$ : viewing, annotating and comparing sequences stored in a relational database. Bioinformatics. 2008;24:2672-6.

36. Hall T. Bioedit, version7.0.0. Distributed by the author. 2004. http://www. mbio.ncsu.edu/BioEdit/bioedit. Accessed 24 December 2015.

37. Bailey TL, Williams N, Misleh C, Li WW. MEME: discovering and analyzing DNA and protein sequence motifs. Nucleic Acids Res. 2006;34:W369-373.

38. Westenberger SJ, Cerqueira GC, El-Sayed NM, Zingales B, Campbell DA, Sturm NR. Trypanosoma cruzi mitochondrial maxicircles display species-and 
strain-specific variation and a conserved element in the non-coding region. BMC Genomics. 2006;7:60.

39. Maslov DA, Avila HA, Lake JA, Simpson L. Evolution of RNA editing in kinetoplastid protozoa. Nature. 1994;368:345-8,

40. Simpson L, Wang SH, Thiemann OH, Alfonzo JD, Maslov DA, Avila HA. Uinsertion/deletion Edited Sequence Database. Nucl Acids Res. 1998;26:170-6.

41. Simpson L. The mitochondrial genome of kinetoplastid protozoa: genomic organization, transcription, replication, and evolution. Ann Rev Microbiol. 1987:41:363-82.

42. Hashimi H, Zimmer SL, Ammerman ML, Read LK, Lukeš J. Dual core processing: MRB1 is an emerging kinetoplast RNA editing complex. Trends Parasitol. 2013;29:91-9.

43. Stuart KD, Schnaufer A, Ernst NL, Panigrahi AK. Complex management: RNA editing in trypanosomes. Trends Biochem Sci. 2005;30:97-105.

44. Flegontov PN, Strelkova MV, Kolesnikov AA. The Leishmania major maxicircle divergent region is variable in different isolates and cell types. Mol Biochem Parasitol. 2006;146:173-9.

45. Borst P, Fase-Fowler F, Hoeijmakers JHJ, Frasch ACC. Variations in maxi-circle and mini-circle sequences in kinetoplast DNAs from different Trypanosoma brucei strains. Biochim Biophys Acta. 1980;610:197-210.

46. Sloof $\mathrm{P}$, de Haan A, Eier W, van lersel M, Boel E, van Steeg $H$, et al. The nucleotide sequence of the variable region in Trypanosoma brucei completes the sequence analysis of the maxicircle component of mitochondrial kinetoplast DNA. Mol Biochem Parasitol. 1992;56:289-99.

47. Horvath A, Maslov DA, Peters LS, Haviernik P, Wüstenhagen T, Kolesnikov AA. Analysis of the sequence of repeats in divergent regions of maxicircular DNA from kinetoplasts of Crithidia oncopelti. Mol Biol (Moscow). 1990;24:1539-48.

48. Flegontov PN, Guo Q, Ren L, Strelkova MV, Kolesnikov AA. Conserved repeats in the kinetoplast maxicircle divergent region of Leishmania sp. and Leptomonas seymouri. Mol Genet Genomics. 2006;276:322-33.

49. Vasil'eva M, Bessolitsina E, Merzlyak E, Kolesnikov A. Identification of the 125 rRNA gene promoter in Leptomonas seymouri mitochondrial DNA. Mol Biol. 2004;38:839-43.

50. Fuglsang A. Distribution of potential type II restriction sites (palindromes) in prokaryotes. Biochem Biophys Res Commun. 2003;310:280-5.

51. Wadkins RM. Targeting DNA, secondary structures. Curr Med Chem. 2000;7:1-15.

52. Higgins CF, McLaren RS, Newbury SF. Repetitive extragenic palindromic sequences, mRNA stability and gene expression: evolution by gene conversion? A review. Gene. 1988;72:3-14.

53. Anez N. Studies on Trypanosoma rangeli Tejera, 1920. IV-A reconsideration of its systematic position. Mem Inst Osw Cruz. 1982;77:405-15.

54. Stevens JR, Teixeira MM, Bingle LE, Gibson WC. The taxonomic position and evolutionary relationships of Trypanosoma rangeli. Int J Parasitol. 1999;29:749-57.

55. Ideozu EJ, Whiteoak AM, Tomlinson AJ, Robertson A, Delahay RJ, Hide G. High prevalence of trypanosomes in European badgers detected using ITS-PCR. Parasit \& Vect. 2015;8:1-6.

56. Lizundia R, Newman C, Buesching CD, Ngugi D, Blake D, Sin YW, et al. Evidence for a role of the host-specific flea (Paraceras melis) in the transmission of Trypanosoma (Megatrypanum) pestanai to the European badger. PLoS One. 2011;6, e16977.

57. Lun ZR, Lai DH, Wen YZ, Zheng LL, Shen JL, Yang TB, et al. Cancer in the parasitic protozoans Trypanosoma brucei and Toxoplasma gondii. Proc Natl Acad Sci U S A. 2015;112:8835-42.

\section{Submit your next manuscript to BioMed Central and we will help you at every step:}

- We accept pre-submission inquiries

- Our selector tool helps you to find the most relevant journal

- We provide round the clock customer support

- Convenient online submission

- Thorough peer review

- Inclusion in PubMed and all major indexing services

- Maximum visibility for your research

Submit your manuscript at www.biomedcentral.com/submit 